\title{
La diversidad biocultural de frutales en huertos familiares de San Andrés Nicolás Bravo, Malinalco, México
}

\author{
The Biocultural Diversity of Fruit Species in Homegardens \\ of San Andrés Nicolás Bravo, Malinalco, Mexico
}

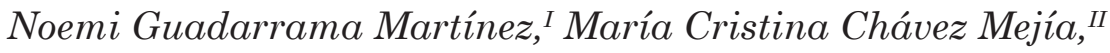 \\ Martín Rubí Arriaga ${ }^{I I I}$ y Laura White Olascoaga ${ }^{I V}$
}

\section{Resumen}

Los huertos familiares son relevantes para la conservación de la biodiversidad, la seguridad alimentaria y la dotación de servicios ecosistémicos. El objetivo de este artículo es analizar la diversidad biocultural en los huertos familiares de San Andrés Nicolás Bravo. Se estudiaron 98 huertos mediante entrevistas a sus dueños sobre su uso y manejo; se identificó la familia, género y especie de las plantas y frutos cultivados; se calculó su abundancia, riqueza, uso e índice de importancia cultural, y se realizó un análisis de sus componentes principales y de clúster para conocer la diferencia entre los huertos. Se identificaron 48 especies con siete usos, donde destacan el alimenticio, medicinal y el destinado a la venta. Además del conocimiento asociado con las diversas formas de aprovechamiento de los frutos, destaca el valor sentimental atribuido a las plantas y su uso ritual. Las especies más abundantes fueron las de mayor valor cultural, por lo que su multifuncionalidad influye en la riqueza de los huertos y muestra la bioculturalidad de las especies frutales. Se concluye que hay diversidad entre los huertos en cuanto a riqueza, forma biológica, abundancia y superficie dedicada a las especies frutales.

\footnotetext{
I Estudiante del Doctorado en Ciencias Agropecuarias y Recursos Naturales de la Universidad Autónoma del Estado de México (UAEM), México. Líneas de interés: etnobotánica, agrodiversidad, desarrollo rural, agroecología. ORCID: 0000-0003-0158-8902. Correo electrónico: urbanoe7@hotmail.com

${ }^{\text {II }}$ Doctorado en Ciencias por la Universidad de East Anglia, Inglaterra. Profesora-investigadora del Instituto de Ciencias Agropecuarias y Rurales de la Universidad Autónoma del Estado de México (UAEM), México. Líneas de interés: agrobiodiversidad, etnobiología, paisajes y territorios campesinos. ORCID: 0000-0003-4337-7572. Correo electrónico: cchavezm@uaemex.mx

III Doctorado en Ciencias Agropecuarias y Recursos Naturales por la Universidad Autónoma del Estado de México (UAEM), México. Profesor-investigador de la Facultad de Ciencias Agrícolas de la Universidad Autónoma del Estado de México (UAEM), México. Líneas de interés: etnobiología, agrobiodiversidad y florística. ORCID: 0000-0001-7547-5017. Correo electrónico: m_rubi65@yahoo.com.mx

IV Doctorado en Ciencias Ambientales por la Universidad Autónoma del Estado de México (UAEM), México. Líneas de interés: florística y ecología de comunidades vegetales naturales y transformadas, particularmente etnobotánica y gestión de procesos sociales. ORCID: 0000-0002-9189-3902. Correo electrónico: laurawhiteo@yahoo.com.mx
} 
Palabras clave: biodiversidad; bioculturalidad; huertos familiares; especies frutales; conservación;

\section{Abstract}

Home gardens are important for biodiversity conservation, food security and ecosystem services. The purpose of this article is to analyze the biocultural diversity of home gardens in San Andrés Nicolás Bravo. Ninety-eight home gardens were studied through interviews with home garden owners on their use and management. The family, genus and species of cultivated plants and fruits were identified; their abundance and index of cultural importance were calculated; and an analysis undertaken of their main components together with a cluster analysis to determine the difference between home gardens. In addition to the knowledge associated with fruit plants, there is an enormous sentimental value attributed to plants and their use in rituals. The most abundant species were those with the greatest cultural value, as a result of which their multifunctionality influences the richness of home gardens and shows the bioculturality of fruit species. The article concludes that there is a diversity between home gardens in terms of the richness, biological forms, abundance and area allocated to fruit species.

Keywords: biodiversity; biocultural; home gardens; fruit species; conservation;

\section{Introducción}

El estudio de la relación sociedad-ambiente es de suma relevancia, ya que la conservación de la biodiversidad depende en gran medida de la cultura. En el 2010 los integrantes del Convenio sobre la Diversidad Biológica (CDB) y la Organización de las Naciones Unidas para la Educación, la Ciencia y la Cultura (UNESCO) lanzaron el Programa sobre la diversidad cultural y biológica, en donde se menciona y promueve el concepto de diversidad biocultural (Maffi y Woodley, 2010 cit. pos. Sabo, 2011; Agnoletti y Rotherham 2015) y se destaca la estrecha relación entre los seres humanos y su ambiente. La diversidad biocultural denota la diversidad de la vida en sus múltiples manifestaciones: biológica, cultural y lingüística, enmarcada en sistemas socioculturales. En este sentido, la diversidad biocultural es una noción dinámica y toma en cuenta los valores y prácticas locales (Elands et al., 2018), así como los conocimientos tradicionales (Cocks y Freerk, 2014).

En la relación sociedad-ambiente, los huertos familiares son reconocidos por la riqueza de especies animales y vegetales que comprende. Se consideran reservorios de biodiversidad relevantes para la seguridad alimentaria, la atención de la salud, entre otros beneficios; así también los huertos familiares son tanto espacios de convivencia familiar y comunitaria, espacios rituales, como lugares para la transmisión de conocimiento ambiental tradicional (Chávez et al., 2017; 
Monroy-Martínez et al., 2016; Cahuich-Campos et al., 2014; Chávez y Vizcarra, 2008). En suma, los huertos familiares son agroecosistemas valiosos para la conservación de la biodiversidad y los sistemas culturales (Calvet et al., 2014; Ibarra et al., 2018).

En el Estado de México los frutos de los huertos familiares se comercializan local y regionalmente; si bien este estado destaca en la producción de frutales (Martínez et al., 2015), los huertos familiares del municipio de Malinalco tienen la peculiaridad de que en ellos se conservan especies frutales relevantes para las economías locales. Así, la disponibilidad de frutos variados a lo largo del año contribuye a la diversidad de la dieta (Guadarrama et al., 2018); de hecho, este municipio es reconocido por la diversidad de especies frutales, ubicándose entre los tres principales centros de comercialización de frutos comestibles no comerciales (SIAP-SAGARPA, 2015).

De acuerdo con Guadarrama (2016), se comercializan a nivel local 59 especies frutales en el tianguis del municipio de Malinalco, las cuales provienen en su mayoría de los huertos de la comunidad de San Andrés Nicolás Bravo; de ahí la relevancia de estudiar esta comunidad en particular. Con el objetivo de contribuir al estudio de la relación entre la biodiversidad biológica y la cultura, además de sumarse a los esfuerzos dirigidos a documentar la diversidad biocultural de los huertos familiares, se realizó el presente estudio, enfocado al análisis de la diversidad biocultural en términos de riqueza, abundancia y uso de especies frutales en los huertos familiares de San Andrés Nicolás Bravo, Malinalco, en el Estado de México.

\section{Comunidad de estudio y métodos}

San Andrés Nicolás Bravo (referido en adelante como San Andrés) pertenece al municipio de Malinalco, Estado de México (Figura 1), tiene una extensión de $217.99 \mathrm{~km}^{2}$ y se encuentra a $99^{\circ} 46^{\prime} 52^{\prime \prime} \mathrm{N}$ y $18^{\circ} 76^{\prime} 77^{\prime \prime}$ O. Cuenta con clima cálido subhúmedo con lluvias en verano; con suelo leptosol y su vegetación corresponde a la de selva baja caducifolia (Torres y Tejero 1998; Rzedowski, 2006). Las especies más representativas en el municipio de Malinalco son el tepeguaje (Lysiloma spp.), el bonete (Jacaratia mexicana), el cazahuate (Ipomoea spp.), el colorín (Erythrina spp.) y el pochote (Ceiba aesculifolia). Su población se compone de 1535 habitantes (772 hombres y 763 mujeres) (Ayuntamiento de Malinalco, 2013). La agricultura de temporal es una de sus actividades principales; cultivan maíz, caña de azúcar y flores de corte. En esta comunidad es recurrente la migración hacia Estados Unidos y también, de forma temporal, al interior del país hacia ciudades como México y Toluca. Los habitantes de San Andrés también se dedican al comercio a nivel local, y para esto aprovechan tanto los frutos de sus huertos como los que recolectan en la selva para comercializarlos en su propia comunidad y en los tianguis municipales de Malinalco, Tenancingo y Tenango. 


\section{Figura 1. Ubicación de San Andrés Nicolás Bravo, Malinalco, México}
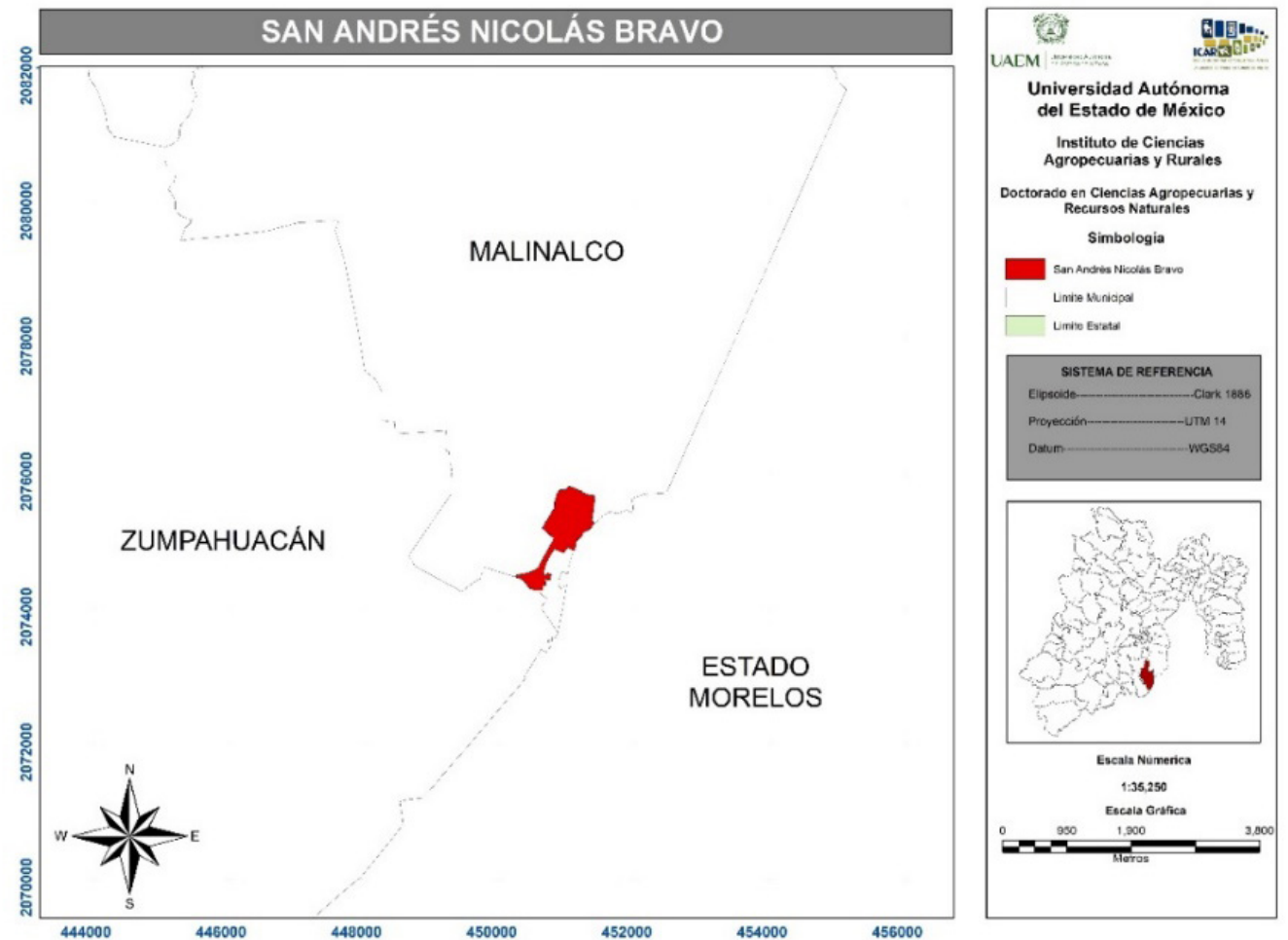

Fuente: elaboración con base en INEGI (2010)

La investigación se realizó de septiembre de 2017 a octubre de 2018 como estudio de caso (Stake, 2000), dado que San Andrés destaca entre otras comunidades del municipio por la diversidad de frutos que se comercializan a nivel local y regional, formando parte del atractivo turístico del municipio, a lo que se suma el hecho de que los frutos son apreciados por ser criollos y orgánicos (Guadarrama, 2016). Se realizaron entrevistas semiestructuradas a 98 propietarios de los huertos (55 mujeres y 43 hombres) sobre el tamaño del huerto, su estructura y manejo, y sobre el aprovechamiento de frutales. Las personas entrevistadas fueron seleccionadas por conveniencia, esto es, por su disposición a participar en el estudio, a través de la técnica de bola de nieve (Goodman, 1961).

Se realizó un inventario de las especies frutales presentes en los 98 huertos familiares, se registró nombre local y usos, además de ser cada una de las especies colectadas y fotografiadas (Manzanares-Medina et al., 2009; Colin et al., 2012), herborizadas e identificadas en el Herbario de la Facultad de Ciencias de la Universidad Autónoma del Estado de México. Asimismo se consultaron las páginas electrónicas World Checklist of Selected Plant Families (WCSP, 2012), The International Plant Names Index (IPNI, 2016), y Tropicos (https://www.tropicos.org/), para conocer o corroborar sus nombres científicos y el origen de las especies frutales. Se considera especie intro- 
ducida a aquella que originalmente no existía en un lugar determinado, mientras que nativa a la que ha evolucionado en un sitio y que está adaptada a las condiciones ecológicas predominantes (Villaseñor y Magaña, 2006; FAO, 2010).

Se usó el Índice de Berger-Parker $(\mathrm{d}=\mathrm{Nmax} / \mathrm{N})$ para calcular la abundancia de la especie o taxón más abundante (Neumann 2001), correspondiendo Nmax al número de individuos del taxón más abundante, y $\mathrm{N}$ al número total de individuos de la muestra. El Índice de Importancia Cultural (Otero, 2005) se empleó para cuantificar los valores de intensidad de uso, frecuencia de mención y valor de uso: IIC $=\Sigma$ (Iuz+Fmz+Vutz), donde IIC equivale al Índice de Importancia Cultural, como la sumatoria de la intensidad de uso, frecuencia de mención y valor de uso; Iuz a la intensidad de uso, considerada como el porcentaje de uso para una especie vegetal "z"; Fmz a la frecuencia de mención, considerada como el porcentaje de menciones para la especie vegetal " $z$ " entre todos los informantes, sin distingos de los usos declarados; y Vutz al valor de uso total para la especie vegetal "z", como la sumatoria de todos los valores de uso de la especie "z" en la categoría de uso "x" de (Vux) a (vun).

Se hicieron dos análisis: uno de componentes principales (ACP) y otro de Clúster (Balzarini et al., 2008), para observar la asociación entre huertos a partir de las variables medidas. En ACP se utilizó el método de Tipologías con las variables especie, forma biológica, abundancia, riqueza (representada por género y especie por huerto) y usos, a partir de la inspección de una matriz de datos para el cálculo de varianzas y correlaciones aproximadas. El Biplot entre los dos primeros componentes principales se hizo con el programa Microsoft Excel (González et al., 2010; Rubí et al., 2014b). El análisis de clúster se realizó con la distancia euclídea promedio y se construyó un dendrograma para el agrupamiento de los huertos según su superficie y riqueza. Se tomaron en cuenta las variables número de huerto, superficie y composición florística de cada uno de ellos y los datos se corrieron en el paquete estadístico PAST (Paleontological Statistics Versión 2.17-).

\section{Resultados}

\section{Riqueza y abundancia de especies frutales}

En los 98 huertos familiares se encontró una riqueza de 48 especies frutales pertenecientes a 38 géneros y 22 familias (Cuadro 1), destacan las familias Rosaceae con siete especies, seguida de Rutaceae con cinco especies y Fabaceae y Myrtaceae con cuatro especies cada una. Las 44 especies reciben un nombre local y cuatro de ellas tienen dos nombres. De las 48 especies, 31 son nativas de México y 17 son introducidas. En cuanto a su forma de vida hay cuatro arbustos (8.3 \%), siete herbáceas (14.58 \%) y 37 árboles (77.08 \%). Respecto a los usos, son siete: alimenticio, para la venta, medicinal, ornamental, ritual, para trueque y aseo personal. Se anota el nombre local y científico de las especies cuando se menciona por primera vez, posteriormente, sólo el nombre local. 
En cuanto a familias más representadas, destaca la Rosaceae como la más importante, lo que coincide con lo reportado por Rubí et al. (2014a) en su estudio sobre huertos del sureste del Estado de México. Otro estudio sobre esa zona White et al. (2017), reporta a la familia Lauraceae y Myrtaceae con mayor número de géneros y especies. En condiciones ambientales similares a las de San Andrés, en la localidad de Coatetelco del estado de Morelos, Sotelo-Barrera et al. (2017) reportan 49 géneros, 65 especies, y 24 familias, siendo las familias Anacardiaceae y Rutaceae las de mayor importancia.

En lo que se refiere a géneros, los estudios de Rubí et al. (2014a y 2014b) en el sureste del Estado de México, sólo reportan 57 y 43 géneros respectivamente para cada estudio, de lo que se esperaría una mayor diversidad a nivel género dado que sus estudios son a nivel región y municipio. En comparación, en San Andrés hay 38 géneros; sólo cinco menos que a nivel región, lo que muestra la importancia de San Andrés en cuanto a la diversidad de sus huertos a nivel género. Citrus es el caso más representativo de acuerdo con los estudios realizados por Pino (2008) en la comunidad de Las Caobas (Gibara, Holguín, Cuba) y con el trabajo de De la Rosa et al. (2014) en huertos familiares de Oaxaca.

Las especies de mayor importancia en San Andrés son el mango (Mangifera indica), lima (Citrus aurantifolia), limón real (Citrus limon), mandarina (Citrus reticulata), tamarindo (Tamarindus indica), guayaba (Psidium guajava), aguacate (Persea americana) y ciruela (Spondias purpurea y Spondias mombin). Salazar-Barrientos et al. (2017), igualmente reportan a cítricos como naranja agria, naranja dulce, limón mexicano, limón persa, mango y mandarina como las principales especies.

Monroy et al. (2016) en un estudio en Xoxocotla, Morelos y López-Ortiz et al. (2017) en Mochicahui, Sinaloa, señalan al limón, la ciruela y el mango como las especies con mayor importancia en los huertos familiares. López-Ortiz et al. (2017) reportan a la ciruela, el aguacate, el limón y la naranja entre las especies de mayor relevancia de huertos en Mochicahui, Sinaloa. Asimismo, Góngora et al. (2016) mencionan al mango y la naranja entre las especies más relevantes en huertos mayas; además de estas plantas, señalan al coco y al plátano como los pilares estructurales de los huertos a nivel península de Yucatán.

Lo anterior muestra que los árboles y otras plantas de porte alto son parte fundamental de los huertos familiares, pues incrementan la diversidad y abundancia de especies y cumplen funciones ambientales, como dar sombra a otras plantas, como por ejemplo los cafetales. Asimismo forman parte del proceso de reciclaje de nutrimentos y sus hojas son materia para elaborar composta, además de servir como refugio para algunos animales, entre otros servicios ambientales (Gutiérrez et al., 2015; Montagnini et al., 2015; White et al., 2017). En San Andrés, los cítricos, el 
nanche (Byrsonima crassifolia) y los mangos dan sombra al café y a plantas ornamentales y condimentarias como la albahaca, el epazote y el orégano. La presencia de árboles en huertos no sólo permite la existencia del huerto como agroecosistema; también son relevantes para que la familia disponga de alimentos en diferentes épocas del año, por ejemplo, Monroy et al. (2016), indican que el 64 \% de las especies arbóreas de los huertos en Morelos, aseguran la alimentación familiar.

Una característica de los huertos familiares es que difieren en su estructura horizontal y vertical, por lo que constituyen sistemas agroforestales complejos (Montagnini et al., 2015), a lo que se añade los factores que contribuyen a la riqueza vegetal, tales como las condiciones climáticas, edáficas y topográficas; las prácticas culturales y el contexto socioeconómico, así como el interés de los propietarios de los huertos en mantener o sustituir las especies que hay en éstos, entre otros factores (Bautista-García et al., 2016). En el caso de San Andrés, si bien hay 48 especies frutales en los 98 huertos estudiados, hay diferencia entre ellos en cuanto a la riqueza, forma biológica y abundancia (Cuadro 1).

\section{Cuadro 1. Familias y especies frutales de 98 huertos familiares de San Andrés, Malinalco, Estado de México}

\begin{tabular}{|c|c|c|c|c|c|c|}
\hline ID & Taxa & Nombre local & $\begin{array}{c}\text { Forma } \\
\text { biológica }\end{array}$ & Origen & Abundancia & $\begin{array}{c}\text { Índice de } \\
\text { Berger/ } \\
\text { Parker }\end{array}$ \\
\hline \multicolumn{7}{|c|}{ Anacardiaceae } \\
\hline $\mathrm{T} 1$ & Mangifera indica $\mathrm{L}$. & Mango & Árbol & I & 240 & 0.10 \\
\hline $\mathrm{T} 2$ & Spondias purpurea L. & Ciruela, jobo & Árbol & $\mathrm{N}$ & 124 & 0.02 \\
\hline T3 & Spondias mombin L. & $\begin{array}{l}\text { Ciruela de bola, } \\
\text { jobo }\end{array}$ & Árbol & $\mathrm{N}$ & 37 & 0.06 \\
\hline \multicolumn{7}{|c|}{ Bignoniaceae } \\
\hline $\mathrm{T} 4$ & Crescentia alata Kunth & Cirian, socos & Árbol & $\mathrm{N}$ & 36 & 0.07 \\
\hline $\mathrm{T} 5$ & $\begin{array}{l}\text { Parmentiera aculeata } \\
\text { (Kunth) Seem. }\end{array}$ & Cuajilote & Árbol & $\mathrm{N}$ & 51 & 0.05 \\
\hline \multicolumn{7}{|c|}{ Bromeliaceae } \\
\hline T6 & Bromelia karatas L. & Timbiriche & Hierba & $\mathrm{N}$ & 63 & 0.04 \\
\hline \multicolumn{7}{|c|}{ Cactaceae } \\
\hline $\mathrm{T} 7$ & Pereskia aculeata Mill. & Uña de gato & Arbusto & $\mathrm{N}$ & 31 & 0.08 \\
\hline \multicolumn{7}{|c|}{ Caricaceae } \\
\hline $\mathrm{T} 8$ & $\begin{array}{l}\text { Jacaratia mexicana A. } \\
\text { DC }\end{array}$ & Bonete & Árbol & $\mathrm{N}$ & 45 & 0.05 \\
\hline T9 & Carica papaya L. & Papaya & Hierba & $\mathrm{N}$ & 68 & 0.04 \\
\hline
\end{tabular}




\begin{tabular}{|c|c|c|c|c|c|c|}
\hline ID & Taxa & Nombre local & $\begin{array}{c}\text { Forma } \\
\text { biológica }\end{array}$ & Origen & Abundancia & $\begin{array}{c}\text { Índice de } \\
\text { Berger/ } \\
\text { Parker } \\
\end{array}$ \\
\hline \multicolumn{7}{|c|}{ Cucurbitaceae } \\
\hline $\mathrm{T} 10$ & Melothria pendula L. & Sandia de ratón & Hierba & $\mathrm{N}$ & 51 & 0.05 \\
\hline \multicolumn{7}{|c|}{ Ebenaceae } \\
\hline $\mathrm{T} 11$ & $\begin{array}{l}\text { Diospyros nigra (J.F. } \\
\text { Gmel.) Perr. }\end{array}$ & Zapote negro & Árbol & $\mathrm{N}$ & 56 & 0.04 \\
\hline \multicolumn{7}{|c|}{ Fabaceae } \\
\hline $\mathrm{T} 12$ & Inga jinicuil Schltdl. & Cuajinicuil & Árbol & $\mathrm{N}$ & 46 & 0.05 \\
\hline $\mathrm{T} 13$ & $\begin{array}{l}\text { Leucaena leucocephala } \\
\text { (Lam.) de Wit }\end{array}$ & Huaje & Árbol & $\mathrm{N}$ & 63 & 0.04 \\
\hline $\mathrm{T} 14$ & Tamarindus indica L. & Tamarindo & Árbol & $\mathrm{I}$ & 67 & 0.04 \\
\hline $\mathrm{T} 15$ & $\begin{array}{l}\text { Pithecellobium dulce } \\
\text { (Roxb.) Benth. }\end{array}$ & Huamuchil & Árbol & $\mathrm{N}$ & 52 & 0.05 \\
\hline \multicolumn{7}{|c|}{ Juglandaceae } \\
\hline $\mathrm{T} 16$ & $\begin{array}{l}\text { Juglans regia L. } \\
\text { Lauraceae }\end{array}$ & Nogal & Árbol & $\mathrm{N}$ & 44 & 0.05 \\
\hline T17 & Persea americana Mill. & Aguacate & Árbol & $\mathrm{N}$ & 58 & 0.04 \\
\hline \multicolumn{7}{|c|}{ Lythraceae } \\
\hline $\mathrm{T} 18$ & $\begin{array}{l}\text { Punica granatum L. } \\
\text { Malpighiaceae }\end{array}$ & Granada roja & Árbol & $\mathrm{N}$ & 37 & 0.06 \\
\hline $\mathrm{T} 19$ & $\begin{array}{l}\text { Byrsonima crassifolia (L.) } \\
\text { Kunth }\end{array}$ & Nanche & Árbol & $\mathrm{N}$ & 37 & 0.06 \\
\hline $\mathrm{T} 20$ & $\begin{array}{l}\text { Malpighia mexicana A. } \\
\text { Juss. }\end{array}$ & $\begin{array}{l}\text { Huachocote, } \\
\text { huachocote }\end{array}$ & Árbol & $\mathrm{N}$ & 39 & 0.06 \\
\hline \multicolumn{7}{|c|}{ Moraceae } \\
\hline $\mathrm{T} 21$ & $\begin{array}{l}\text { Artocarpus heterophyllus } \\
\text { Lam. }\end{array}$ & Yaca & Árbol & I & 25 & 0.10 \\
\hline \multicolumn{7}{|c|}{ Musaceae } \\
\hline $\mathrm{T} 22$ & Musa spp. L. & Plátano & Hierba & $\mathrm{N}$ & 27 & 0.09 \\
\hline \multicolumn{7}{|c|}{ Myrtaceae } \\
\hline $\mathrm{T} 23$ & Psidium guajava L. & Guayaba blanca & Árbol & $\mathrm{N}$ & 107 & 0.02 \\
\hline $\mathrm{T} 24$ & Psidium montanum Mill. & Guayaba rosa & Árbol & $\mathrm{I}$ & 50 & 0.05 \\
\hline $\mathrm{T} 25$ & $\begin{array}{l}\text { Psidium sartorianum (O. } \\
\text { Berg) Nied. }\end{array}$ & $\begin{array}{l}\text { Arrayán, } \\
\text { guayabilla }\end{array}$ & Árbol & $\mathrm{N}$ & 36 & 0.07 \\
\hline $\mathrm{T} 26$ & \begin{tabular}{|l} 
Syzygium jambos (L.) \\
Alston
\end{tabular} & Poma rosa & Árbol & $\mathrm{N}$ & 25 & 0.10 \\
\hline \multicolumn{7}{|c|}{ Oxilidaceae } \\
\hline $\mathrm{T} 27$ & Averrhoa carambola L. & Carambolo & Árbol & $\mathrm{I}$ & 29 & 0.08 \\
\hline
\end{tabular}




\begin{tabular}{|c|c|c|c|c|c|c|}
\hline ID & Taxa & Nombre local & $\begin{array}{c}\text { Forma } \\
\text { biológica }\end{array}$ & Origen & Abundancia & $\begin{array}{c}\text { Índice de } \\
\text { Berger/ } \\
\text { Parker }\end{array}$ \\
\hline T28 & Passiflora edulis Sims & Maracuyá & Hierba & $\mathrm{I}$ & 48 & 0.05 \\
\hline T29 & $\begin{array}{l}\text { Passiflora tarminiana } \\
\text { Coppens \& V.E. Barney }\end{array}$ & Granada & Hierba & $\mathrm{I}$ & 37 & 0.06 \\
\hline T30 & Passiflora ligularis Juss. & $\begin{array}{l}\text { Granada de } \\
\text { moco }\end{array}$ & Hierba & $\mathrm{N}$ & 37 & 0.06 \\
\hline \multicolumn{7}{|c|}{ Rosaceae } \\
\hline T31 & Crataegus mexicana DC. & Tejocote & Árbol & $\mathrm{I}$ & 53 & 0.05 \\
\hline T32 & $\begin{array}{l}\text { Eriobotrya japonica } \\
\text { (Thunb.) Lindl. }\end{array}$ & Níspero & Árbol & $\mathrm{I}$ & 18 & 0.13 \\
\hline T33 & Malus domestica Borkh & Manzana & Árbol & $\mathrm{N}$ & 30 & 0.08 \\
\hline T34 & Morus nigra L. & Mora & Arbusto & $\mathrm{I}$ & 33 & 0.07 \\
\hline T35 & Prunus persica (L). Batch & Durazno criollo & Árbol & $\mathrm{I}$ & 46 & 0.05 \\
\hline T36 & $\begin{array}{l}\text { Prunus serotina Ehrh. } \\
\text { subsp. Capuli (Cav.) } \\
\text { McVaugh }\end{array}$ & Capulín & Árbol & I & 45 & 0.05 \\
\hline T37 & Rubus fruticosus L. & Zarzamora & Arbusto & $\mathrm{I}$ & 56 & 0.04 \\
\hline \multicolumn{7}{|c|}{ Rubiaceae } \\
\hline T38 & Morinda citrifolia $\mathrm{L}$. & Nonis & Arbusto & $\mathrm{I}$ & 25 & 0.10 \\
\hline \multicolumn{7}{|c|}{ Rutaceae } \\
\hline T39 & $\begin{array}{l}\text { Casimiroa edulis La } \\
\text { Llave }\end{array}$ & Zapote blanco & Árbol & $\mathrm{N}$ & 37 & 0.06 \\
\hline $\mathrm{T} 40$ & $\begin{array}{l}\text { Citrus aurantifolia } \\
\text { Swingle }\end{array}$ & Lima & Árbol & I & 75 & 0.03 \\
\hline $\mathrm{T} 41$ & Citrus limon (L.) Osbeck & Limón real & Árbol & $\mathrm{N}$ & 48 & 0.05 \\
\hline $\mathrm{T} 42$ & Citrus medica L. & Cidra & Árbol & $\mathrm{I}$ & 36 & 0.07 \\
\hline $\mathrm{T} 43$ & Citrus reticulata Blanco. & Mandarina & Árbol & $\mathrm{N}$ & 74 & 0.03 \\
\hline \multicolumn{7}{|c|}{ Salicaceae } \\
\hline $\mathrm{T} 44$ & $\begin{array}{l}\text { Xylosma flexuosa (Kunth) } \\
\text { Hemsl. }\end{array}$ & Huismarines & Árbol & $\mathrm{N}$ & 37 & 0.06 \\
\hline \multicolumn{7}{|c|}{ Sapotaceae } \\
\hline $\mathrm{T} 45$ & $\begin{array}{l}\text { Pouteria sapota (Jacq.) } \\
\text { H.E. Moore \& Stearn }\end{array}$ & Mamey & Árbol & $\mathrm{N}$ & 23 & 0.10 \\
\hline $\mathrm{T} 46$ & Chrysophyllum cainito L. & Caimito & Árbol & $\mathrm{I}$ & 84 & 0.03 \\
\hline $\mathrm{T} 47$ & $\begin{array}{l}\text { Manilkara zapota (L.) P. } \\
\text { Royen }\end{array}$ & Chico zapote & Árbol & $\mathrm{N}$ & 44 & 0.05 \\
\hline $\mathrm{T} 48$ & $\begin{array}{l}\text { Pouteria campechiana } \\
\text { (Kunth) Baehni }\end{array}$ & Zapote borracho & Árbol & $\mathrm{N}$ & 33 & 0.07 \\
\hline
\end{tabular}

Nota: Nativa de México (N) e introducida (I); ID de cada especie en el Biplot (T).

Fuente: elaboración propia. 
A través del caso de San Andrés puede verificarse que la conservación de las especies está directamente relacionada con su multifuncionalidad: la riqueza y abundancia de los huertos de esta comunidad se debe en gran medida a la apropiación material y simbólica de las especies ahí cultivadas como lo reportan para otros casos Toledo (2013) y Cocks y Freerk (2014). Así, el análisis de componentes principales (ACP) (Figura 2) muestra que los dos primeros componentes (CP1 y CP2), explican el 79 \% de la variación presente. El componente principal 1 (CP1), contribuye con el $55 \%$, y el componente principal 2 (CP2) con el $24 \%$. Las variables abundancia, presencia (en cuántos huertos se encuentra cada especie) y usos explican la mayor variación asociada al CP1.

\section{Figura 2. Biplot para representar la relación entre forma biológica, abundancia, riqueza y usos de las especies frutales de San Andrés Nicolás Bravo, Malinalco}

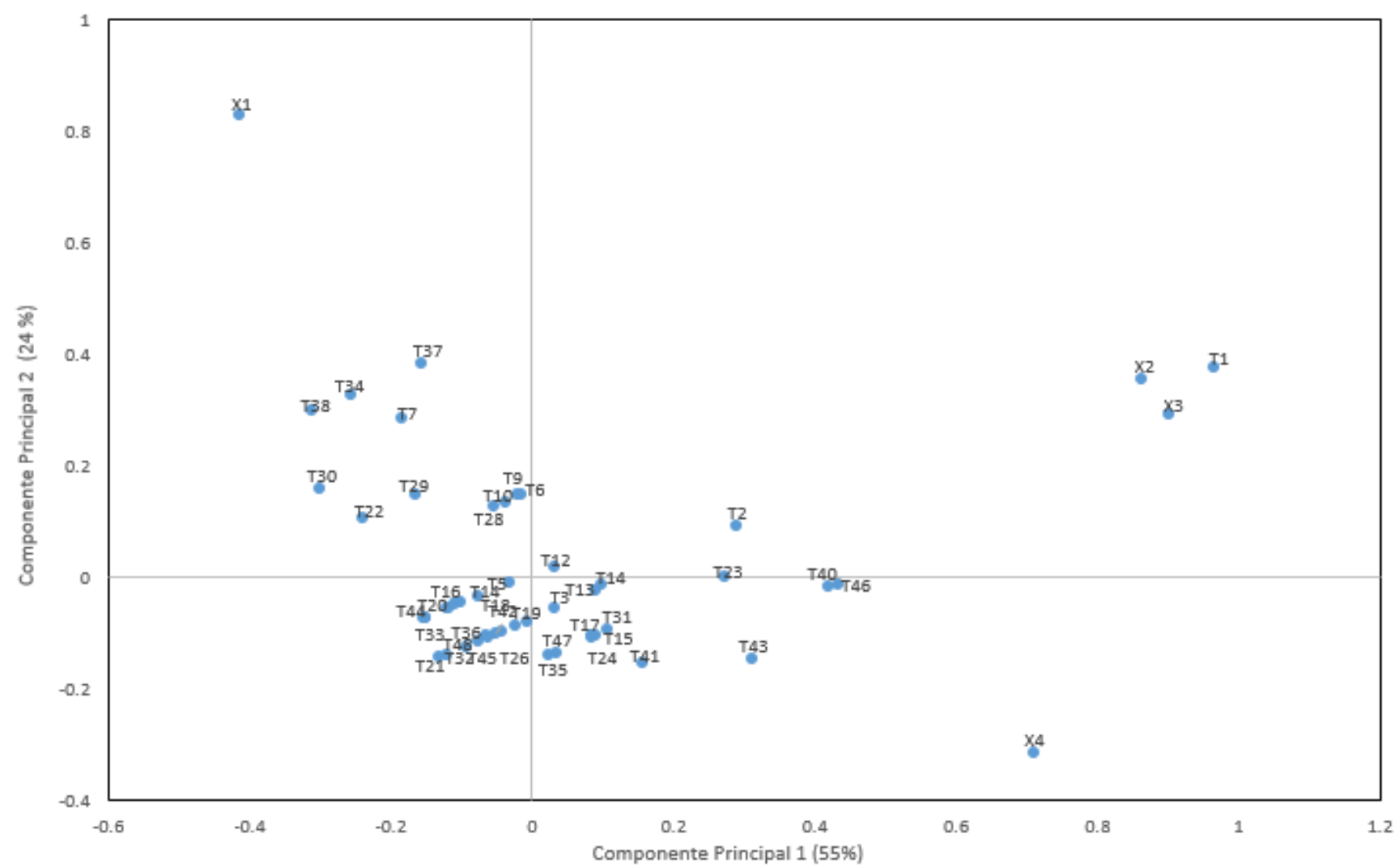

Nota: Las especies frutales se identifican con T1...Tn y las variables evaluadas se representan con V1...V4. El número asignado a las especies corresponde al que se indica en la Tabla 1 (T1-T48). A las variables evaluadas se les asignaron los prefijos. V1: Forma biológica, V2: Abundancia, V3: Presencia en HF, V4: Usos.

Fuente: elaboración propia. 


\section{Socjedad Ambiente}

En el CP1 las familias botánicas más representativas en cuanto a riqueza de los huertos son: Rutaceae, Fabaceae, Myrtaceae y Anacardiaceae, que se encuentran representadas en el CP1, aquí se ubican entre otras: lima (Citrus aurantifolia, T40), huaje (Leucaena leucocephala), guayaba blanca y mango. Estas cuatro especies tienen una abundancia promedio de 107 individuos y están presentes en el $51 \%$ de los huertos familiares. Las familias, géneros y especies del cuadrante 1 son las que se asocian con abundancia y presencia. En el cuadrante 4 del CP1 están las familias Fabaceae, Lauraceae, Myrtacea y Rutacea con huamúchil (Pithecellobium dulce), aguacate, guayaba rosa (Psidium montanum) y mandarina (Citrus reticulata), respectivamente; en promedio hay 61 individuos de estas especies. Si bien hay similitud con las familias del cuadrante 1, las especies presentes en el cuadrante 4 se relacionan con el número de usos de cada especie.

La forma biológica (V1) fue la variable con mayor representatividad en el CP2 y está relacionada con las familias Bromeliaceae, Cactaeae y Rosaceae, esta última con siete especies y seis géneros. Entre las especies que se encuentran en este CP2 están: timbiriche (Bromelia karatas), uña de gato (Pereskia aculeata), papaya (Carica papaya), mora (Morus nigra) y zarzamora (Rubus fruticosus). Destaca que en el cuadrante 3 del CP2 se agrupan las especies arbóreas.

Uno de los factores que influyen en la abundancia de plantas frutales en San Andrés es el manejo del huerto para la multiplicación de especies por medio de semilla, esqueje y plantación. Por ejemplo, el mango, el tamarindo, la ciruela, el cuajinicuil (Inga jinicuil), el caimito (Chrysophyllum cainito), el aguacate y los cítricos son propagados a través de semilla; mientras que el huachocote (Malpighia mexicana), el arrayán (Psidium sartorianum), la papaya y la poma rosa (Syzygium jambos), por semilla o esqueje.

La construcción de un dendrograma permitió organizar a los huertos familiares en tres grupos principales a una distancia de corte de 50 aproximadamente (Figura 3); los principales elementos que crearon estos grupos fueron: superficie donde se encuentran las especies frutales, forma biológica, y riqueza y abundancia por huerto familiar. El primer grupo representa el $12.24 \%$ de los 98 huertos y cuenta con una superficie promedio de $200 \mathrm{~m}^{2}$ y una riqueza promedio de 17 especies frutales por $\mathrm{HF}$.

En este grupo las familias botánicas más representativas son Fabaceae, que se encuentra presente en 11 de los $12 \mathrm{HF}$, con huaje, tamarindo y huamúchil; Pasifloraceae con granada (Passiflora tarminiana) y granada de moco (Passiflora ligularis); seguida de Anacardiaceae, presente en 10 de los HF, con mango y ciruela de bola; Myrtaceae presente en 10 HF con guayaba blanca y guayaba rosa. La forma biológica más representativa son los árboles y las herbáceas, presentes en el $91.6 \%$ de los HF de este grupo, seguidos por los arbustos, presentes en el $75 \%$ de los HF. 
Figura 3. Dendrograma mediante el análisis de clúster entre

la superficie, riqueza y abundancia de las especies frutales de los 98 huertos familiares de San Andrés Nicolás Bravo, Malinalco

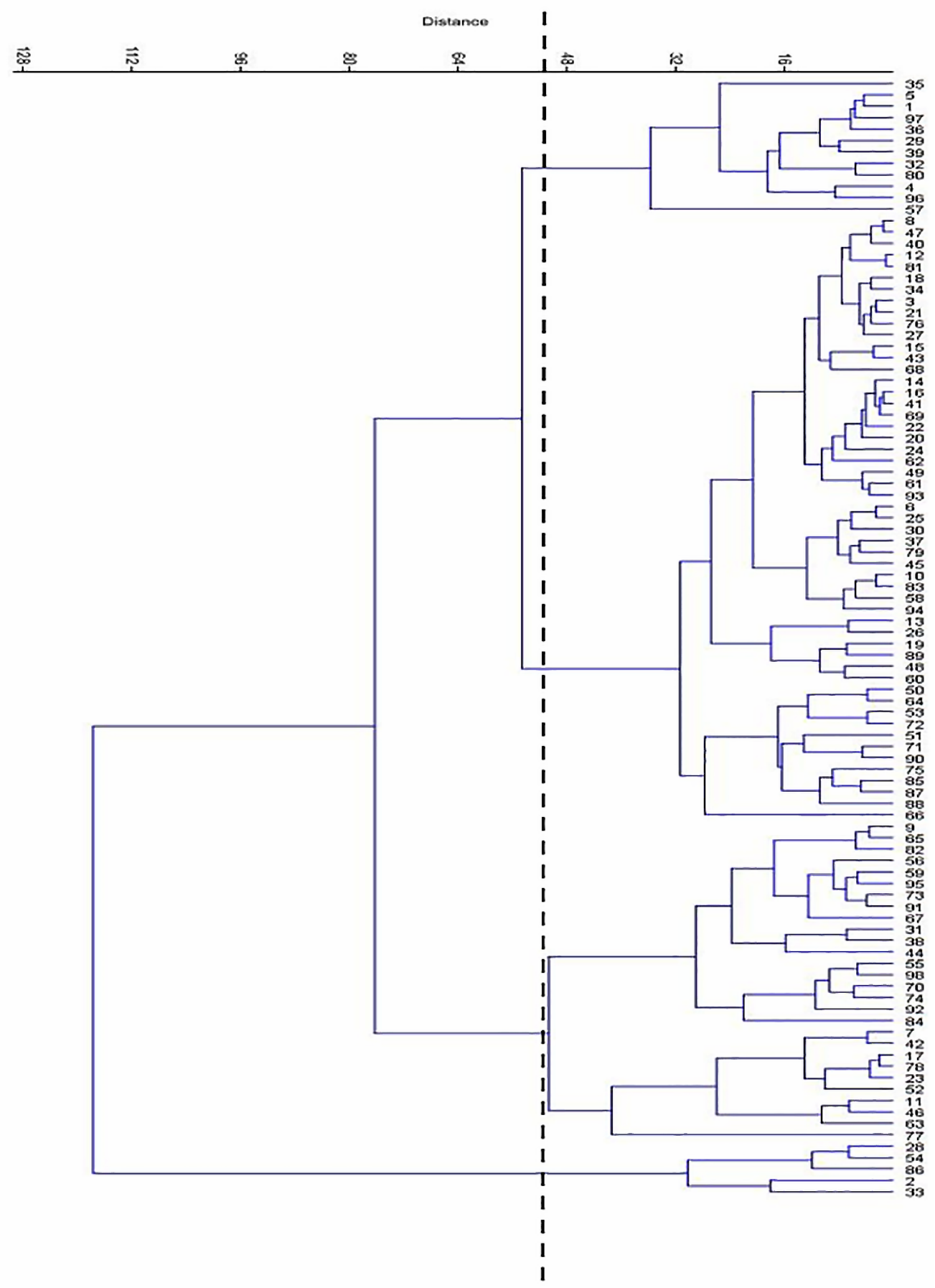

Fuente: elaboración propia. 
El grupo dos representa el $54.09 \%$ de los $98 \mathrm{HF}$, cuentan con una superficie promedio de $205 \mathrm{~m}^{2}$ y una riqueza promedio de 20 especies frutales por HF. En este grupo las familias botánicas más representativas son Rutaceae, con zapote blanco, lima, cidra y mandarina; seguida de Anacardiaceae, presente en 52 de los $\mathrm{HF}$, con mango, ciruela y ciruela de bola; Cucurbitaceae, presente en $52 \mathrm{HF}$, con sandía de ratón (Melothria pendula); Myrtaceae, presente en 50 HF con guayaba blanca y guayaba rosa, arrayán; y Fabaceae en 48 de los $53 \mathrm{HF}$, con huaje, tamarindo y huamúchil (Pithecellobium dulce). En cuanto a la forma biológica, la más representativa son los árboles, que se encuentran en $98.11 \%$ de los HF de este grupo, seguidos de las herbáceas, presentes en $81.13 \%$ de estos HF, y por último los arbustos, presentes en $60.37 \%$ de los HF.

El tercer y último grupo lo conforman el $33.67 \%$ de los $98 \mathrm{HF}$, que cuentan con una superficie promedio de $202 \mathrm{~m}^{2}$, y un promedio de 18 especies frutales por HF. La familia Anacardiaceae se encuentra presente en 30 de los HF, con mango, ciruela y ciruela de bola; Rutaceae, presente en 27 de los HF, con las especies zapote blanco, lima y mandarina; Myrtaceae, presente en $26 \mathrm{HF}$ con guayaba blanca y arrayán; Cactaceae presente en $23 \mathrm{HF}$ con la especie Pereskia aculeata (uña de gato, T7). La forma biológica más representativa son los árboles, presentes en el $93.93 \%$ de los HF de este grupo, seguido de los arbustos, presentes en el 81.81 \%, y por último las herbáceas, con el $72.72 \%$ de presencia en los HF.

La relación entre la superficie dedicada a las especies frutales y su riqueza y forma biológica muestra la necesidad de atender las implicaciones que la fragmentación de los huertos tiene sobre la conservación de la biodiversidad y por tanto del conocimiento asociado con su aprovechamiento, en tanto parte de los sistemas culturales que los sostienen. González-Jácome (2012), advierte sobre la pérdida de huertos en México por su fragmentación y por el cambio de uso del suelo.

\section{Usos de especies frutales y su importancia cultural}

La importancia de una especie crece en relación con sus diversas formas de aprovechamiento, es decir, con su carácter multiusos. En los huertos estudiados se encontró que el 100 \% de las especies son alimenticias y para la venta; seguidos por los usos medicinal (95.8\%), ritual (54.1\%), ornamental (37.5 \%), y en menor proporción para el aseo personal (6.25\%) y para trueque (6.25 \%). La suma de los porcentajes es más de 100 debido a que todas las especies reciben de dos a siete usos: una especie tiene dos usos; trece especies, tres; diecinueve, cuatro usos; ocho especies, cinco; tres especies, seis, y sólo una especie recibe siete usos (Cuadro 3). 


\section{Uso alimenticio}

Todas las especies frutales son consumidas en fresco, pero también hay diferentes formas de preparación. Como por ejemplo, con la lima, mandarina y limón real también se elaboran licores, nieves, ensaladas, conservas y jugos, o son consumidos como fruta cristalizada. El aguacate destaca como alimento en fresco o para la elaboración de ensaladas, nieves y otros alimentos; el arrayán y el mango se consumen como fruta fresca agregándole limón y chile piquín, o también se preparan con ellos licores, jaleas y nieves. Monroy-Martínez et al. (2016), también reportan que las especies frutales (29 de 45) son relevantes para la alimentación de los habitantes de Pueblo Nuevo, Morelos, México.

\section{Especies utilizadas para la venta}

Todas las especies son ofertadas los días miércoles y domingos en el tianguis de Malinalco, de acuerdo con su disponiblidad por temporada del año; para su comercialización se presentan en platos, montones, racimos, canastas o en bolsas de plástico (para detalle ver Guadarrama, 2016). Algunos frutos como la yaca, el caimito, la uña de gato, la sandía de ratón, el huachocote y el timbiriche son apreciados por visitantes y turistas por considerarlos exóticos. La gente local igualmente compra frutos de los huertos, y los valora porque los consideran como "fruta criolla" y orgánica, debido a que se tiene entendido que no se usan agroquímicos para su cultivo. García-Flores et al. (2016), en su estudio sobre huertos en el sureste del Estado de México indican que los frutos importantes para la venta en esta región son el aguacate, el mamey y las anonas.

\section{Especies destinadas al trueque}

Al final del día, cuando concluyen las ventas, los comerciantes de frutos realizan trueque con huaje, guayaba rosa, mandarina, tamarindo y tejocote, los cuales son intercambiados principalmente por jitomate, tomate, leña y pan. De acuerdo con los entrevistados, hay frutos que no se intercambian, como el mango, el arrayán, la poma rosa, el bonete (Jacaratia mexicana) y la yaca (Artocarpus heterophyllus). El mango no se intercambia porque una manera de conservar esta especie en la comunidad es guardando la semilla para poder reproducir la especie; el arrayán tampoco se intercambia porque es un fruto escaso debido a que hay pocos árboles en la comunidad (36 ejemplares); en cuanto a la poma rosa, debido a que se puede conservar por más tiempo, prefieren guardarla para su venta en el siguiente día de tianguis de la cabecera municipal; el bonete (Jacaratia mexicana) no se intercambia porque es perecedero; y por último, la yaca no se intercambia debido a que es escasa en la comunidad (25 ejemplares), es un fruto grande y vale más que otros frutos, alrededor de 50 pesos, y tendría que ser intercambiado por ropa, pero esta práctica no ocurre en el tianguis de Malinalco. 
De acuerdo con García-Flores et al. (2016) en Malinalco se intercambia níspero, aguacate, guayaba, limón, mango y plátano; en San Andrés se intercambian también seis especies, pero el mango no, contrario a lo reportado por estos autores. En la comunidad de Nenelá, Yucatán, el huerto aporta $60.8 \%$ de los productos paa el trueque, entre ellos papaya, plátano, guayaba, tamarindo, anonas, cítricos y ciruela (Lope-Alzina, 2014).

\section{Uso medicinal}

Son empleadas para prevenir, aliviar o curar ciertos padecimientos y enfermedades, algunas eliminan la sed o se usan como tónicos, como el huachocote, la papaya, el tejocote (Crataegus mexicana), el zapote blanco y la cidra; también se utilizan para tratar dolor estomacal, infección de vías urinarias y gripes. El mango, el tamarindo, el aguacate y la guayaba se usan para la eliminación de parásitos intestinales, bajar la inflamación de estómago y tratar dolores de cabeza, riñón, hígado, vientre y ovarios. El arrayán es valorado para tratar afecciones respiratorias; su extracto es un ingrediente utilizado en enjuagues bucales, y también se preparan cremas y pomadas para tratar dolores musculares y para hidratar la piel.

Algunas otras se utilizan para tratamiento de síndromes de filiación cultural como la guayaba combinada con zapote blanco, bonete y nanche, utilizados para tratar el espanto de los niños (desde recién nacidos hasta los 13 años de edad). Con dichas plantas los bañan y posteriormente les colocan alcohol en el cuerpo y los visten de color rojo para quitarles el susto. Cuando a las personas "les da aire", para aliviarse o bien para prevenir su padecimiento, se juntan en racimo plantas como el soco (Crescentia alata), la uña de gato, el nogal (Juglans regia) y el huamúchil para "hacerse una limpia”, pasando el racimo por el cuerpo. El mango en la medicina local es usado en infusiones, cataplasmas y en gotas. Se usa también para tratar empacho, dolor de estómago, y enfermedades de vías urinarias y respiratorias; la gente comenta que su uso es muy efectivo para aliviar estos malestares.

El uso medicinal de las plantas de los huertos es relevante, por ejemplo, Álvarez-Quiroz et al. (2017), reportan que en Ayapa, Tabasco, la gente utiliza 140 especies. En San Andrés, doce de ellas también son medicinales: aguacate, caimito, carambola, chicozapote, ciruela, guayaba, limón, mango, nanche, noni, papaya y tamarindo. En cuanto al centro de México, Sánchez-Alejo et al., (2016), señalan la importancia de las plantas medicinales para la comunidad de San Jerónimo Boncheté, Estado de México.

\section{Uso ritual}

Diversidad de plantas son utilizadas como ofrenda para las casas, para hacer ramos de limpias y con ellas aliviar malestares espirituales, asimismo son usadas en los fandangos y en los inicios 
de siembras o de cosechas. Por ejemplo, en su uso ritual en la agricultura, frutos como la lima, la mandarina, el limón y el aguacate son utilizados para formar un círculo en medio del terreno antes de la siembra, adentro del cual se posicionan las personas para pedir por una buena cosecha. Se cree que si no se hace esta ofrenda el 15 de mayo, que es el día de San Isidro, la yunta podría dañar los cultivos al momento de la escarda. Cahuich-Campos et al. (2014) también muestran la relación entre el huerto y el cultivo de las parcelas agrícolas en el ejido X-Mejía en Campeche; relación manifiesta en las prácticas de petición y agradecimiento que se realizan a través de la preparación de comida ritual, en la que el huerto aporta el $54 \%$ de los ingredientes, tales como mandarina, naranja, plátano y papaya.

En San Andrés, cuando se divide un huerto para establecer uno nuevo en donde habitará otra familia, se acostumbra colocar en el terreno recién demarcado una ofrenda de frutas y una cruz hecha de hojas de maíz y tallos de caña de azúcar. Mediante una oración se pide para que haya prosperidad y abundancia para la nueva familia, y a la entrada del huerto se colocan especies frutales como arrayán, aguacate, poma rosa, mango, níspero, entre otras. Las especies frutales son elegidas de acuerdo con los gustos e intereses de la nueva familia que establecerá su propio huerto, el cual está destinado a proporcionarles alimento diario y a ser fuente de ingresos económicos.

\section{Uso ornamental}

Entre las especies frutales utilizadas para este fin se puede mencionar a la lima, la mandarina y el limón real, que son utilizados en adornos y centros de mesa en fiestas comunitarias y familiares, de los cuales se aprovecha específicamente el fruto y las hojas. La ciruela, el arrayán, el níspero (Eriobotrya japonica) y la poma rosa se utilizan en arreglos florales elaborados con prácticamente toda la planta (rama, hojas y fruta) para ocasiones festivas. En el tianguis municipal destaca su venta debido a que se consideran fruta exótica. En otros estudios se señala el uso ornamental de las especies de los huertos; por ejemplo, en La Encrucijada, Tabasco, las plantas ornamentales son las segundas en importancia entre las especies registradas por Bautista-García et al. (2016); en el caso de San Andrés resalta el uso de frutos con fines ornamentales. En huertos de San Juan Atzingo, municipio de Ocuilan, Estado de México, el 58 \% de las especies son de uso ornamental.

\section{Especies usadas para el aseo personal}

El jugo de la lima, del limón y el limón real son usados como desodorante y para arreglar el cabello, esto es, para fijarlo. Al igual que en el cuidado de la salud, para el aseo personal la gente prefiere hacer uso de los recursos con los que cuentan y, de acuerdo con lo recogido en el estudio, se busca "en lo mínimo usar químicos", por lo que son valorados los cítricos para el arreglo personal. Debido 
a sus usos y formas diversas de aprovechamiento, las plantas frutales son comercializadas a lo largo del año de acuerdo con la época de crecimiento y desarrollo de cada especie. Rubí et al. (2014b), también menciona el uso de especies frutales para el aseo personal en el municipio de Sultepec, Estado de México.

\section{Valor de importancia cultural}

En el Índice de Importancia Cultural (IIC) (Cuadro 2), las especies de mayor valor fueron el limón real (76.04), la lima (61.42), la mandarina (60.47) y el mango (51.37). De los cítricos, el limón real tiene seis usos, y se encontraron 48 individuos en 17 huertos. Es el menos abundante de la familia Rutaceae y el que menos se comercializa, pero es importante para el trueque y forma parte de la diversidad de cítricos en los huertos. La importancia cultural del mango se relaciona con sus usos varios: es de destacar que además de aprovecharse como fruta fresca e ingrediente de platillos locales, se comercializan sus hojas y ramas, utilizadas en la medicina tradicional y en arreglos florales. El mango como fruto también es importante por uso ornamental, al formar parte de los arreglos para centros de mesa en fiestas patronales y familiares. En cuanto a su venta, es un fruto valorado, que se encuentra con frecuencia en el tianguis de Malinalco. Esta especie se encuentra en el $66.32 \%$ de los huertos estudiados.

La apropiación material y simbólica de las plantas influye en la conservación e introducción de especies en los huertos. Si bien dicha apropiación forma parte de tradiciones milenarias, continúa existiendo debido a la dinámica en su manejo (Toledo, 2013; Moctezuma, 2013; Pulido-Salas et al., 2017). En San Andrés, las plantas introducidas (17 especies) obtuvieron el mayor índice de importancia cultural, tales como el mango, el tamarindo, las granadas y los cítricos; no obstante que los habitantes de esta comunidad las han hecho ya parte de su vida diaria, su número es menor frente al de las especies nativas, que suman 31 .

Hallazgos similares a los de este estudio respecto al lugar preponderante que el mango y los cítricos tienen en los huertos, se encuentran en los trabajos de Salazar-Barrientos et al. (2017) sobre Yucatán, así como el de Monroy et al. (2016) sobre los huertos de Pueblo Nuevo, Morelos, el de Góngora et al. (2016) en comunidades mayas, y el de López-Ortiz et al. (2017). El valor e importancia del mango en San Andrés es de 73.01 y se encuentra en $66.3 \%$ de los 98 huertos; casos similares son expuestos también por García-Flores (2016) en su estudio sobre otras comunidades del sureste del Estado de México, y por Montoya (2011) en el trabajo que realizó en la comunidad de Tlayacapan, Morelos, donde las especies con mayor abundancia son el mango, el limón y la ciruela. 
Cuadro 2. Usos e importancia cultural de especies frutales en San Andrés Nicolás Bravo, Malinalco, Estado de México, México

\begin{tabular}{|c|c|c|c|c|c|}
\hline Nombre común & Usos & $\begin{array}{l}\text { Valor de uso } \\
\text { total por porcen- } \\
\text { taje (Vutz) }\end{array}$ & $\begin{array}{l}\text { Frecuencia } \\
\text { de mención } \\
(\text { Fmz })\end{array}$ & $\begin{array}{l}\text { Intensi- } \\
\text { dad de } \\
\text { usos (Iuz) }\end{array}$ & $\begin{array}{l}\text { Índice de } \\
\text { valor cultural } \\
\text { IIC }\end{array}$ \\
\hline Limón real & $1,2,3,4,5,6$ & 68.03 & 4.82 & 3.19 & 76.04 \\
\hline Lima & $1,2,3,4,5,6$ & 53.29 & 4.94 & 3.19 & 61.42 \\
\hline Mandarina & $1,2,3,4,5,6,7$ & 52.34 & 4.94 & 3.19 & 60.47 \\
\hline Mango & $1,2,3,4,5$ & 41.65 & 7.06 & 2.66 & 51.37 \\
\hline Tamarindo & $1,2,3,4,5,7$ & 27.72 & 4.71 & 2.66 & 35.09 \\
\hline Guayaba blanca & $1,2,3,4,5$ & 24.16 & 4.71 & 2.66 & 31.53 \\
\hline Ciruela & $1,2,3,4$ & 23.14 & 5.41 & 2.13 & 30.68 \\
\hline Zapote borracho & $1,2,3,4$ & 13.64 & 3.06 & 2.13 & 18.82 \\
\hline Durazno criollo & $1,2,3,4,5$ & 13.71 & 2.09 & 2.66 & 18.46 \\
\hline Huamuchil & $1,2,3,4,5$ & 13.31 & 2.05 & 2.66 & 18.02 \\
\hline Caimito & $1,2,3,4$ & 12.06 & 2.31 & 2.13 & 16.49 \\
\hline Guayaba rosa & $1,2,3,4,5$ & 11.60 & 1.84 & 2.66 & 16.09 \\
\hline Chico zapote & $1,2,3,4,5$ & 11.17 & 2.24 & 2.66 & 16.07 \\
\hline Arrayán & $1,2,3,4$ & 10.81 & 2.12 & 2.13 & 15.06 \\
\hline Ciruela de bola & $1,2,3$ & 10.43 & 2.82 & 1.60 & 14.85 \\
\hline Aguacate & $1,2,3,4,5$ & 10.48 & 1.65 & 2.66 & 14.79 \\
\hline Timbiriche & $1,2,3,4$ & 10.19 & 2.35 & 2.13 & 14.67 \\
\hline Cidra & $1,2,3,4$ & 10.46 & 1.88 & 2.13 & 14.47 \\
\hline Poma rosa & $1,2,3,4$ & 10.39 & 1.88 & 2.13 & 14.40 \\
\hline Sandía de ratón & $1,2,3,4$ & 10.60 & 1.53 & 2.13 & 14.26 \\
\hline Mamey & $1,2,3,5$ & 9.37 & 1.88 & 2.13 & 13.38 \\
\hline Tejocote & $1,2,3,4,5$ & 8.84 & 1.41 & 2.66 & 12.91 \\
\hline Uña de gato & $1,2,3,4$ & 8.82 & 1.79 & 2.13 & 12.73 \\
\hline Capulín & $1,2,3,5$ & 8.55 & 1.65 & 2.13 & 12.32 \\
\hline Papaya & $1,2,3,5$ & 8.42 & 1.65 & 2.13 & 12.20 \\
\hline Bonete & $1,2,3$ & 7.60 & 2.09 & 1.60 & 11.29 \\
\hline Maracuyá & $1,2,3,4$ & 7.30 & 1.41 & 2.13 & 10.84 \\
\hline Zapote blanco & $1,2,3,5$ & 7.03 & 1.53 & 2.13 & 10.69 \\
\hline Nogal & $1,2,5$ & 7.66 & 1.32 & 1.60 & 10.57 \\
\hline Yaca & $1,2,3,4$ & 6.98 & 1.32 & 2.13 & 10.42 \\
\hline Nanche & $1,2,3,5$ & 6.76 & 1.41 & 2.13 & 10.30 \\
\hline Carambolo & $1,2,3$ & 6.49 & 1.79 & 1.60 & 9.87 \\
\hline
\end{tabular}




\begin{tabular}{|l|l|l|l|l|l|}
\hline Nombre común & Usos & $\begin{array}{l}\text { Valor de uso } \\
\text { total por porcen- } \\
\text { taje (Vutz) }\end{array}$ & $\begin{array}{l}\text { Frecuencia } \\
\text { de mención } \\
\text { (Fmz) }\end{array}$ & $\begin{array}{l}\text { Intensi- } \\
\text { dad de } \\
\text { usos (Iuz) }\end{array}$ & $\begin{array}{l}\text { Indice de } \\
\text { valor cultural } \\
\text { IIC }\end{array}$ \\
\hline Manzana & $1,2,5$ & 7.15 & 1.06 & 1.60 & 9.80 \\
\hline Zapote negro & $1,2,3,5$ & 6.14 & 1.32 & 2.13 & 9.59 \\
\hline Cirian, socos & $1,2,3$ & 5.00 & 1.41 & 1.60 & 8.01 \\
\hline Níspero & $1,2,3,5$ & 4.68 & 1.01 & 2.13 & 7.82 \\
\hline Cuajilote & $1,2,3$ & 4.92 & 1.29 & 1.60 & 7.81 \\
\hline Huismarines & $1,2,3$ & 4.86 & 1.29 & 1.60 & 7.75 \\
\hline Huaje & $1,2,3,7$ & 4.24 & 1.13 & 1.60 & 6.96 \\
\hline Zarzamora & $1,2,3$ & 4.04 & 1.06 & 1.60 & 6.69 \\
\hline Granada roja & $1,2,3$ & 3.96 & 1.04 & 1.60 & 6.60 \\
\hline Nonis & $1,2,3$ & 3.85 & 1.04 & 1.60 & 6.49 \\
\hline Cajinicuil & $1,2,3$ & 3.87 & 0.94 & 1.60 & 6.40 \\
\hline Granada & $1,2,3$ & 3.39 & 0.89 & 1.60 & 5.88 \\
\hline Mora & $1,2,3$ & 3.16 & 0.82 & 1.60 & 5.57 \\
\hline Huachocote & $1,2,3$ & 3.00 & 0.80 & 1.60 & 5.40 \\
\hline Plátano & $1,2,3$ & 2.66 & 0.71 & 1.60 & 4.96 \\
\hline Granada de moco & 1,2 & 2.08 & 0.52 & 1.06 & 3.66 \\
\hline
\end{tabular}

Nota. Usos : comestible (1); venta (2); medicinal (3); ritual (4), ornamental (5); y aseo personal (6).

Fuente: elaboración propia.

El tamarindo se usa en la medicina tradicional para tratar dolor estomacal y empacho; en la cocina local para la elaboración de licores, dulces, nieves, ensaladas, salsas, entre otros. Para venta y trueque, se encuentra presente en el tianguis la mayor parte de los meses del año. Su uso ornamental forma parte de las ofrendas en las iglesias durante festividades comunitarias y familiares; para estas ocasiones se llenan canastas de tamarindo, lo que para ellos significa abundancia. La ciruela de alto IIC tiene cuatro usos: comestible, medicinal, ornamental y para la venta (comercializada localmente, es uno de los pocos frutos con los que los vendedores realizan el truque en el tianguis semanal de Malinalco). Posteriormente se encuentra la guayaba blanca, con valor de 0.04, muy cercano al de la ciruela, de la cual hay dos tipos de acuerdo con el color de su pulpa: la blanca y la rosa. Aunque ambas tienen cinco usos, la guayaba blanca es la que se utiliza con mayor frecuencia, quizás porque es más dulce que la rosa, por esa razón se encuentra un mayor número de individuos en los huertos.

El caimito, papaya, timbiriche y huaje tienen cuatro usos, siendo el más importante el alimenticio; en cuanto a la venta, el timbiriche y el caimito son considerados especies exóticas pues 
no son conocidos en el tianguis de Malinalco, y en este sentido resultan atractivos para los compradores, sobre todo para los turistas. En el caso de la lima, y de acuerdo con su IIC, recibe seis usos, lo que indica la importancia que tiene en la vida cotidiana y festiva de las familias de San Andrés.

En cuanto al zapote borracho, el durazno y el huamúchil, dos de éstos son especies nativas y tienen cuatro o cinco usos, siendo el de mayor relevancia el alimenticio. Por ejemplo, al huamúchil se le da uso ornamental, como por ejemplo en arreglos florales. En la medicina es apreciado para el tratamiento posparto: con éste y otras plantas, la partera baña a la mujer después de haber dado a luz. El caimito, la guayaba rosa y el chico zapote tienen entre 16 y 17 de valor de importancia cultural. El fruto del caimito es de un sabor ácido y tiene poca vida poscosecha, sin embargo, los habitantes de San Andrés lo consideran importante para los rituales pues de acuerdo con sus creencias, al colocarlo en las ofrendas del ritual agrícola permite alejar el mal y obtener buenas cosechas.

El arrayán, la ciruela de bola, el aguacate, el timbiriche y la cidra tienen un valor de importancia cultural de entre 14 y 15 . El aguacate tiene cinco usos: alimentario, medicinal, para la venta, ornamental y ritual; a pesar de su importancia cultural, sólo está en el $14.28 \%$ de los huertos; esto se deba quizás a que de acuerdo con la gente, el aguacate requiere más cuidados que otras especies, dado que es susceptible a plagas y enfermedades, por lo que para evitarlas hacen podas de saneamiento y fertilizan con composta. Solamente es comercializado o intercambiado en la comunidad y no en el tianguis de Malinalco.

De acuerdo con Zimik et al. (2012), la diversidad de flora en los huertos se debe a varios factores, tanto ambientales como sociales, siendo uno de ellos el manejo. A este respecto, en San Andrés la diversidad es resultado del cuidado y fomento de especies tanto nativas como introducidas, cada una de las cuales satisfacen necesidades particulares. Por ejemplo, la gente planta árboles de yaca, mango y cuajinicuil cerca de la casa y los cítricos en los límites del huerto familiar, es decir también funcionan como linderos.

Gracias al manejo y aprovechamiento del huerto, en San Andrés hay 31 especies nativas y 17 introducidas; resultados similares reportan White et al. (2017) y Rubí et al. (2014b), quienes indican que $54 \%$ de las especies reportadas en su estudio son nativas. Rubí et al. (2014a) reportan en otro estudio que $59 \%$ son nativas. Por su parte, Sotelo-Barrera et al. (2017) reportan $55 \%$ de especies nativas, mientras que López-Ortiz et al. (2017) documentan que 31 especies de las 59 encontradas en su estudio son introducidas. Igualmente, De la Rosa et al. (2014) hacen mención que más del $50 \%$ de las especies en los huertos estudiados son introducidas. En el caso de San Andrés, no obstante que las especies de mayor índice de valor cultural son introducidas (10 especies), predominan las especies nativas; lo cual quizás se deba a que en los huertos familiares se toleran y fomentan plantas que forman parte de la flora local, y también debido al manejo y aprovechamien- 


\section{Socjedad Ambiente}

to de los huertos; por ejemplo, en el deshierbe no se remueven todas las plantas, además de que se usan especies nativas en la alimentación y medicina tradicional.

Es de resaltar que, en medio de las especies frutales, la gente cultiva maíz, nopales, plantas medicinales, condimentarias y ornamentales, además de tolerar e incluso fomentar arbustos y hierbas que crecen de forma silvestre a partir de mecanismos naturales de dispersión, los cuales reciben varios usos. Es por esto que algunos huertos se asemejan a terrenos agrícolas, como lo apuntan Montagnini et al. (2015). Las especies frutales son también valoradas de acuerdo con el trabajo requerido para mantenerlas en buen estado; la gente señala que hay plantas que requieren mucha atención, como el aguacate, ya señalado anteriormente, y otras que requieren de poco cuidado. Por ejemplo, el mango no requiere de muchos cuidados; de acuerdo con los testimonios recogidos "si un árbol se cuida demasiado deja de producir y ya no sirve, por eso es mejor no cuidarlo tanto", sólo cuando "les hacen ojo", "se les coloca una cabeza de ajo cerca del tronco para que vuelvan a producir".

Cabe destacar que la gente valora a los mangos y a los cítricos por ser parte de la historia de los huertos que establecieron sus abuelos y padres. En este sentido son considerados como producto del trabajo de sus antecesores y como parte importante del espacio en el que día a día llevan a cabo sus actividades, guardando así un valor sentimental. Las especies que en particular guardan este valor sentimental son el mango, los cítricos (especialmente el limón real), la yaca, el níspero y el maracuyá. El aprecio por plantas que fueron establecidas por familiares de los dueños de los huertos también es reportado por Chávez-García et al. (2012), asimismo mencionan que algunas especies son plantadas para proteger contra envidias y hechizos. El estudio de Chávez-García et al. en el ejido Francisco I. Madero en Tabasco y el presente sobre San Andrés, muestran los valores intangibles atribuidos a las plantas, en tanto parte de la diversidad biocultural de los huertos.

De acuerdo con la propuesta de la diversidad biocultural (Maffi, 2001) se destaca que, en San Andrés, todas las especies reciben un nombre local, y cuatro tienen dos nombres; en otros casos, no todas las especies reciben nombre local, como lo reportan Monroy y García (2013) en huertos de Xoxocotla, Morelos. En cuanto a los usos (que son siete) de las especies frutales en San Andrés, es de resaltar que son todas alimenticias y las mismas son valoradas para la venta en el tianguis municipal. Las diversidad biocultural permite tener acceso a una amplia variedad de alimentos; como lo apunta Rodríguez (2012), la flora de los huertos es significativa para la gastronomía local. También es de destacar que $95.5 \%$ de las especies son medicinales, lo que muestra su relevancia para la salud en San Andrés y la práctica de la medicina tradicional.

$\mathrm{Al}$ respecto de las plantas medicinales de los huertos, White et al. (2013), en San Nicolás, municipio de Malinalco registran 36 especies; 24 son frutales, y de ellas, 23 son de uso medicinal. 
También en San Andrés destacan los árboles: 38 de las 48 especies frutales; si bien el presente estudio se enfoca en los frutos, los árboles son relevantes para la medicina tradicional, ya que, a la sombra de ellos, mujeres y hombres conocedores de las plantas medicinales llevan a cabo sus prácticas curativas (Chávez et al., 2017).

El valor cultural de cada especie se relaciona directamente con los usos asignados, lo cual permite ubicar a las especies frutales en la categoría de uso múltiple. El conocimiento local desempeña un papel importante en la identificación de plantas frutales, además de contribuir a la formación de alternativas para las estrategias de vida de los habitantes de la comunidad, en función de la conservación, la protección del medio ambiente y la economía local, como lo documentan también Ángel et al. (2017). Así, la multifuncionalidad de las especies de los huertos, al igual que el manejo y el valor sentimental atribuido a las plantas, reflejan la diversidad biocultural que se fundamenta en el conocimiento local para el establecimiento, manejo y aprovechamiento del huerto, como lo señalan Calvet et al. (2014) y Cano et al. (2016). En suma, la diversidad biocultural de los huertos en San Andrés es fuente importante de su vida cotidiana y ritual.

En San Andrés, la diversidad de los huertos en cuanto a la riqueza y abundancia de las especies frutales que en ellos crece, junto con los siete usos identificados para su aprovechamiento, se relacionan con el conocimiento local, lo cual deriva en la conservación no sólo de la biodiversidad, sino también de prácticas culturales como la medicina tradicional, los rituales para el cultivo de la tierra y el trueque. Además el valor sentimental atribuido al algunas especies influye en la biodiversidad de los huertos.

\section{Conclusiones}

El presente estudio sobre las especies frutales de San Andrés es una contribución al análisis de la diversidad biocultural de un sistema milenario como lo es el huerto familiar. La riqueza de las especies frutales en los huertos de San Andrés está representada por 22 familias botánicas, 38 géneros y 48 especies. Los huertos son diversos en cuanto a riqueza de especies frutales; esta biodiversidad se relaciona con la forma biológica, superficie de los huertos y los usos que reciben las plantas. Asimismo, la abundancia de las especies frutales se relaciona con su manejo y aprovechamiento, por lo que las plantas de mayor importancia cultural son más abundantes. De este modo, esta riqueza biológica corresponde a los usos varios de las especies frutales presentes en la vida cotidiana y ritual de la comunidad. 


\section{Agradecimientos}

Al Consejo Nacional de Ciencia y Tecnología (CONACYT), por la beca otorgada a la primera autora para la realización del Doctorado en Ciencias Agropecuarias y Recursos Naturales, dentro del proyecto de investigación "Recursos alimentarios locales y seguridad alimentaria en comunidades campesinas del Estado de México”, registrado en la Universidad Autónoma del Estado de México. A todas las personas de la comunidad de San Andrés Nicolás Bravo, Malinalco, que colaboraron y nos compartieron información sobre sus huertos familiares y nos transmitieron sus conocimientos. A los doctores Gerardo Rodríguez Ortiz, Andrés Gonzales Huerta y Andrés Xingú López por la asesoría para la realización e interpretación de los análisis estadísticos y al Dr. Jaime Mejía Carranza por sus valiosos comentarios al artículo.

\section{Referencias}

Álvarez-Quiroz, Violeta; Caso-Barrera, Laura; Aliphat-Fernández, Mario; Galmiche-Tejeda, Ángel (2017). "Plantas medicinales con propiedades frías y calientes en la cultura zoque de Ayapa, Tabasco, México". Boletín latinoamericano y del caribe de plantas medicinales y aromáticas, 16(4), pp. 428-454.

Agnoletti, Mauro y Rotherham, Ian (2015). "Landscape and Biocultural Diversity: Emerging Paradigms in Conservation and Planning". Biodiversity and Conservation, 24, pp. 3155-3165. doi: 10.1007/s10531-015-1003-8

Ángel, Yimi; Pimentel, Maggy, y Suárez, Juan (2017). "Importancia cultural de vegetación arbórea en sistemas ganaderos del municipio de San Vicente del Caguán, Colombia”. Revista U.D.C.A Actualidad and Divulgación Científica, 20(2), pp. 393-401.

Argumedo, Alejandro (S/F). "Territorios bioculturales indígenas. Una propuesta para la protección de territorios indígenas y el buen vivir". Recuperado de http://www.internationalfunders.org/ documents/TerritoriosBioculturalesIndigenas.pdf (última consulta 29 de agosto de 2018).

Ayuntamiento de Malinalco (2013) "Plan de Desarrollo Municipal Malinalco, México 2013-2015". Recuperado de https://www.ipomex.org.mx/recursos/ipo/files_ipo/2013/47/11/598ab8b9160eafd7264dfc38976eaa2a.pdf

Balzarini, Mónica; González, Laura Alicia; Tablada, Margot; Casanoves, Fernando; Di Rienzo, Julio Alejandro, y Robledo, Carlos W. (2008). "Infostat. Manual del usuario”. Córdoba: Editorial Brujas.

Bautista-García, Germán; Sol-Sánchez, Ángel; Velázquez-Martínez, Alejandro y Llanderal-Ocampo, Tangaxuhan (2016). "Composición florística e importancia socioeconómica de los huertos familiares del ejido La Encrucijada, Cárdenas, Tabasco". Revista Mexicana de Ciencias Agrícolas, 14(15), pp. 2725-2740. 
Cahuich-Campos, Diana; Huicochea Gómez, Laura, y Mariaca Méndez, Ramón (2014). "El huerto familiar, la milpa y el monte maya en las prácticas rituales y ceremoniales de las familias de X-Mejía, Hopelchén, Campeche”. Relaciones. Estudios de historia y sociedad, 35(140), pp. $157-184$.

Calvet, Laura; Garnatje, Teresa; Parada, Montserrat; Valles, Joan, y Reyes, Victoria (2014). "Más allá de la producción de alimentos: los huertos familiares como reservorio de diversidad biocultural". Revista Ambienta, 107, pp. 40-53.

Cano, Margarita; De la Tejera, Beatriz; Casas, Alejandro; Salazar, Lourdes, y García, Raúl (2016). "Conocimientos tradicionales y prácticas de manejo del huerto familiar en dos comunidades tlahuicas del Estado de México, México". Revista de la Red Iberoamericana de Economía Ecológica, 25, pp. 81-94. Recuperado de https://ddd.uab.cat/record/159752

Capote, Maricela; Pérez, Yesenia; Rodríguez, A.; Sánchez, O, y Sánchez, Y. (2008). "Inventario de la composición frutícola en un huerto familiar como indicador de la diversidad vinculada al mejoramiento participativo". Memorias del XVI Congreso del INCA. La Habana, Cuba. DIE, p. 8.

Chávez, Cristina y Vizcarra, Ivonne (2008). "El solar familiar y sus relaciones de género". Sociedades rurales, producción y medio ambiente, 10(15), pp. 41-70.

Chávez, María; White, Laura; Moctezuma, Sergio, y Herrera, Francisco (2017). "Prácticas curativas y plantas medicinales: un acercamiento a la etnomedicina de San Nicolás, México". Cuadernos Geográficos, 56(2), pp. 26-47.

Chávez-García, Elsa; Rist, Stephan, y Galmiche-Tejeda, Ángel (2012). "Lógica de manejo del huerto familiar en el contexto del impacto modernizador en Tabasco, México". Cuadernos de desarrollo rural, 9(68), pp. 177-200.

Cocks, Michelle Linda y Wiersum, Freerk (2014). "Reappraising the Concept of Biocultural Diversity: A Perspective from South Africa". Human Ecology, 42, pp.727-737.

Colín, Hortencia; Hernández, Andrea y R. Monroy, Rafael (2012). "El manejo tradicional y agroecológico en un huerto familiar de México, como ejemplo de sostenibilidad". Etnobiología 10(2): $12-28$.

De la Rosa, Perla; Vásquez, Marco; Villegas, Yuri, y Jerez, Martha (2014). "Los huertos familiares y la seguridad alimentaria de Cuilapam de Guerrero, Oaxaca, México". Revista de Agroecosistemas, 1(1), pp. 40-51.

Elands, Birgit; Vierikko, Kati; Andersson, Erik; Fischer, Leonie; Goncalves, Paula; Haase, D.; Kowarik, Ingo; Luz, Ana; Niemela, Jary; Santos, Margarida, y Wiersum, Freerk (2018). "Biocultural Diversity: A Novel Concept to Assess Human-Nature Interrelations, Nature Conservation and Stewardship in Cities". Urban Forestry \& Urban Greening, 32, pp. 102-116. doi: https://doi.org/10.1016/j.ufug.2018.04.006.

FAO (2010). "Evaluación de los recursos forestales mundiales 2010. Términos y definiciones". Roma, Italia: Programa de Evaluación de los Recursos Forestales-FAO, Documento de trabajo 144. Recuperado de http://www.fao.org/3/am665s/am665s00.pdf 
García-Flores, José; Gutiérrez-Cedillo, Jesús; Balderas-Plata, Miguel, y Araújo-Santana, María (2016). "Estrategia de vida en el medio rural del altiplano central mexicano: el huerto familiar". Agricultura, sociedad y desarrollo, 13(4), pp. 621-641. Recuperado de http://www.scielo. org.mx/pdf/asd/v13n4/1870-5472-asd-13-04-00621.pdf

Góngora, Ricardo; Flores, Salvador; Ruenes, María; Aguilar, Wilian, y García, Jesús (2016). "Uso tradicional de la flora y fauna en los huertos familiares mayas en el municipio de Campeche, Campeche, México", 3(9), pp. 379-389. Recuperado de http://www.scielo.org.mx/pdf/era/ v3n9/2007-901X-era-3-09-00379.pdf.

González-Jácome, Alba (2012). "Del huerto a los jardines y vecindades: procesos de cambio en un agroecosistema de origen antiguo". En Ramón Mariaca (ed.), El huerto familiar del sureste de México. Villahermosa, Tabasco: SERNAPAM/ECOSUR, pp. 487-521.

González, Andrés: Pérez, Delfina; Sahagún, José; Franco, Omar; Morales, E.J.; Rubí, Martín; Gutiérrez, Francisco, y Balbuena, Artemio (2010). "Aplicación y comparación de métodos univariados para evaluar la estabilidad en maíces del valle Toluca-Atlacomulco, México". Agronomía costarricense, 34, pp. 129-143.

Goodman, Leo (1961). "Snowball Sampling”. Annals of Mathematical Statistics, 32(1), pp. 148-170. doi: 10.1214 / aoms / 1177705148

Guadarrama, Noemi; Sangerman-Jarquín, Dora; Chávez, Cristina; Rubí, Martín (2018). "Estrategias de comercialización de los frutos en el tianguis de Malinalco, México". Revista mexicana de ciencias agrícolas, 9(4), pp. 841-9. Recuperado de https://cienciasagricolas.inifap.gob.mx/ editorial/index.php/agricolas/article/view/1400.

Guadarrama, Noemi (2016). "Venta de frutos en el tianguis de Malinalco, Estado de México. Propuesta para su conservación y diversificación de usos". (Tesis de Maestría en Agroindustria Rural, Desarrollo Territorial y Turismo Agroalimentario). México: Universidad Autónoma del Estado de México.

Guerra, Verónica y Ochoa, Susana (2006). "Evaluación espacio-temporal de la vegetación y uso del suelo en la reserva de la biósfera Pantanos de Cetla, Tabasco (1990-2000)". Investigaciones geográficas, 59, pp. 7-25.

Gutiérrez Cedillo, Jesús; White, Laura; Juan, José y Chávez, María (2015). "Agro ecosistemas de huertos familiares en el subtrópico del altiplano mexicano. Una visión sistémica”. Tropical and Subtropical Agroecosystems, 18(3), pp. 237-250.

Ibarra, José; Caviedes, Julián; Barreau, Antonia, y Pessa, Natalia (2018). Huertas familiares y comunitarias. Cultivando soberanía alimentaria. Santiago de Chile: Universidad Católica de Chile.

INEGI. Instituto Nacional de Geografía Estadistica y Geoinformática (2010) Marco Geoestadístico Municipal Versión 5.0, México: INEGI.

IPNI (International Plant Names Index) (2016). “The International Plant Names Index". Recuperado de http://www.ipni.org (última consulta febrero de 2018). 
Lascurain, Maite; Avendaño, Sergio; Del Amo, Silvia, y Niembro, Aníbal (2010). "Guía de frutos silvestres comestibles en Veracruz". Ciudad de México: Fondo CONACYT-CONAFOR/ INECOL, $142 \mathrm{pp}$.

Lope-Alzina, Diana (2014). "Una red comunal de acceso a alimentos: el huerto familiar como principal proveedor de productos para intercambio en una comunidad maya yucateca". Gaia Scientia, 8, pp. 199-215. Recuperado de file:///C:/Users/523-33/Downloads/2014.Lope-Alzina. GAIA.Huertosredessociales.pdf

López-Ortíz, Demetrio; Osuna, Israel; De la Torre, Mayra, y Olivos, Aramís (2017). "Diversidad de árboles frutales de traspatio en Mochicahui, El Fuerte, Sinaloa, México". Revista Biodiversidad Neotropical, 7(1), pp. 6-13. doi: http://dx.doi.org/10.18636/bioneotropical.v7i1.489

Maffi, Luisa (2001). "Linking Language and Environment: A Co-Evolutionary Perspective". En C. L. Crumley (ed.), New Directions on Anthropology and Environment: Intersections. California: Altamira Press, pp. 24-48.

Manzanares-Medina Gladys; Flores-Martínez Alejandro y Hunn Eugene (2009). "Los huertos familiares zapotecos de San Miguel Talea de Castro, Sierra Norte de Oaxaca, México". Etnobiología 7:191-228.

Martínez, Isabel; Rubí, Martín; González, Andrés; Pérez, Delfina; Franco, Omar, y Castañeda, Álvaro (2015). "Frutos y semillas comestibles en el Estado de México". Revista Mexicana de Ciencias Agrícolas, 6(2), pp. 331-346.

Moctezuma, Sergio (2013). "Cambios y continuidades en el manejo de huertos familiares del suroeste de Tlaxcala, México”. Perspectivas Latinoamericanas, 10(1), pp. 83-101. Recuperado de https://nanzan-u.repo.nii.ac.jp.

Monroy, Rafael y García, Alejandro (2013). "La fauna silvestre con valor de uso en los huertos frutícolas tradicionales de la comunidad indígena de Xoxocotla, Morelos, México". Revista Etnobiología, 11(1), pp. 44-52.

Monroy-Martínez, Rafael; Ponce-Díaz, Alma; Colín-Bahena, Hortensia; Monroy-Ortiz, Columba; García-Flores, Alejandro (2016). "Los huertos familiares tradicionales soporte de seguridad alimentaria en comunidades campesinas del estado de Morelos, México". Ambiente y Sostenibilidad, 6, pp. 33-43.

Montagnini, Florencia; Somarriba, Eduardo; Murgueitio, Enrique; Fassola, Hugo y Eibl, Beatriz (eds.) (2015). Sistemas agroforestales: funciones productivas, socioeconómicas y ambientales. Cali, Colombia: CIPAV/Turrialba; Costarrica: CATIE, 454 pp. Recuperado de https://drflorenciamontagnini.wordpress.com/montagnini-et-al-2015-spanish-agroforestry-book/.

Montoya, Mirna (2011). "Los huertos tradicionales de Tlayacapan, Morelos: productores de bienes y servicios ambientales con significado cultural" (Tesis de licenciatura). Cuernavaca, Morelos: Facultad de Ciencias Biológicas-Universidad Autónoma del Estado de Morelos, 196 pp.

Neumann, Marcus y Starlinger, Franz (2001). "The Significance of Different Indices for Stand Structure and Diversity in Forest". Forest Ecology and Management, 145, pp. 91-106. 
Ochoa, Juan y Ladio, Ana (2015). "Current Use of Wild Plants with Edible Underground Storage Organs in a rural Population of Patagonia: Between Tradition and Change". Journal of Ethnobiology and Ethnomedicine, (11), pp. 1-14. doi: https://doi.org/10.1186/s13002-015-0053-z

Organización de las Naciones Unidas (2010). "Convenio sobre la biodiversidad biológica”, 32 pp.

Otero, Roberto (2005). “Árboles nativos de usos múltiples y sistemas agroforestales tradicionales en el municipio de Acapulco de Juárez, Guerrero" (Tesis de maestría). Texcoco, México: Universidad Autónoma Chapingo, $181 \mathrm{pp}$.

Pino, María (2008). "Diversidad agrícola de especies de frutales en el agroecosistema campesino de la comunidad de las Caobas, Gibara, Holguín”. Revista Cultivos Tropicales, 29(2), pp. 5-10.

Pulido-Salas, María; Ordóñez, María, y Cálix, Héctor (2017). “Flora, usos y algunas causales de cambio en quince huertos familiares en el municipio de José María Morelos, Quintana Roo, México". Península, 12, pp.119-145.

Rodríguez, Silvia (2012). "El despojo de la riqueza biológica: de patrimonio de la humanidad a recurso bajo soberanía del Estado”. México: Itaca, 332 pp.

Rubí, Martín; Martínez, Isabel; González, Andrés; Pérez, Delfina; Cruz, Juan, y Guadarrama, Noemi (2014a). "Catálogo de especies frutales presentes en el sureste del Estado de México, México”. Revista Mexicana Ciencias Agrícolas, 5(8), pp. 1509-1517.

Rubí, Martín; González, Andrés; Martínez, Isabel; Franco, Omar; Ramírez, José; López, José, y Hernández, Guadalupe (2014b). "Inventario de especies frutales y aspectos etnobotánicos en Sultepec, Estado de México”. Revista Internacional de Botánica Experimental, 83(1), pp. 203-211.

Rzedowski, Jerzy (2006). Vegetación de México. México: Comisión Nacional para el Conocimiento y Uso de la Biodiversidad, 505 pp.

Sabo, Autumn (2011). Revisión del libro Biocultural Diversity Conservation: A Global Sourcebook. Luisa Maffi and Ellen Woodley 2010. Ecological Restoration, 29(3), pp. 315-316. https://www. muse.jhu.edu/article/448475.

Salazar-Barrientos, Lucia; Magaña, Miguel, y Latournerie, Luis (2017). "Importancia económica y social de la agrobiodiversidad del traspatio en una comunidad rural de Yucatán, México”. Agricultura, Sociedad y Desarrollo, 12(1), pp. 1-14. Recuperado de http://www.scielo.org.mx/ $\mathrm{pdf} / \mathrm{asd} / \mathrm{v} 12 \mathrm{n} 1 / \mathrm{v} 12 \mathrm{n} 1 \mathrm{a} 1 . \mathrm{pdf}$.

Sánchez-Alejo, Rocío; Rangel-Villafranco, Mónica; Cristóbal-Sánchez, Griselda; Martínez-García, Aurora, y Pérez-Mondragón, María (2016). "Sistematización del conocimiento tradicional asociado al uso de las plantas medicinales en una comunidad mazahua". Revista Iberoamericana de Ciencias. Recuperado de file://C:/Users/HP\%200454088/Documents/SAN\%20JERONIMO\%20BONCHETE\%20conocimiento\%20tradicional.pdf

SIAP-SAGARPA (Servicio de Información Agroalimentaria y Pesquera - Secretaria de Agricultura, Ganadería, Desarrollo Rural, Pesca y Alimentación) (2015). "Servicio de Información Agroalimentaria y Pesquera”. México: SAGARPA (última consulta 15 de enero de 2018). 
Sotelo-Barrera, Mireya; García, Edmundo; Romero, Angélica; Monroy, Rafael, y Luna, Mario (2017). "Arboreal Structure and Cultural Importance of Traditional Fruit Homegardens of Coatetelco, Morelos, Mexico". Revista Chapingo Serie Ciencias Forestales y del Ambiente, 23(1), pp. 137-153. doi: 10.5154/r.rchscfa.2016.01.002

Stake, Robert (2000). "Case Studies". En N. K. Denzin e Yvonna S. Lincoln (eds.), Handbook of Qualitative Research, Thousand Oaks, CA: Sage Publications, pp. 134-164.

Toledo, Víctor Manuel (2013). "El metabolismo social: una nueva teoría socioecológica”. Relaciones, (136), pp. 41-71. Recuperado de http://www.scielo.org.mx/pdf/rz/v34n136/v34n136a4.pdf

Torres, María y Tejero, Daniel (1998). "Flora y vegetación de la Sierra de Sultepec, Estado de México". Anales del Instituto de Biología Serie Botánica, 69, pp. 135-174.

Tropicos.org. Missouri Botanical Garden (https://www.tropicos.org)

Villaseñor, José y Magaña, Patricia (2006). "Plantas introducidas en México". Ciencias, pp. 38-40.

White, Laura; Juan, José; Chávez, María, y Gutiérrez, José (2013). "Flora medicinal en San Nicolás, municipio de Malinalco, Estado de México”. Revista Polibotánica, 35, pp. 173-206.

White, Laura; Chávez, María, y Mondragón, David (2017). "Análisis del estrato arbóreo de agroecosistemas en una zona de transición ecológica”. Ecosistemas Recursos Agropecuarios, 4(11), pp. 255-264. doi: http://dx.doi.org/10.19136/era.a4n11.882

WCSP (World Checklist of Selected Plant Families). Facilitado por Royal Botanic Gardens, Kew (2012). Recuperado de http://apps.kew.org/wcsp/home.do (última consulta noviembre de 2017).

Zimik, Link; Saikia, Purabim y Khan, Mohammed (2012). "Comparative Study on Homegardens of Assam and Arunachal Pradesh in Terms of Species Diversity and Plant Utilization Pattern". Research Journal of Agricultural Sciences, 3, pp. 611-618.

Editora asociada: Esperanza Tuñón Pablos Sometido: 8 de septiembre de 2019 Aceptado: 4 de febrero de 2020 Publicado: 12 de mayo de 2020 